\title{
Construction of a Simple USB Powered LED Light Source for Illuminating Optical Microscopes
}

\author{
Ayomide Oluwaseyi Agunloye ${ }^{1, *}$, Emmanuel Idowu Agunloye ${ }^{2}$, Nathaniel Oluwadusi Ajayi ${ }^{1}$ \\ ${ }^{1}$ Department of Physics and Electronics, Adekunle Ajasin University, Akungba-Akoko, Nigeria \\ ${ }^{2}$ Ebman Biomedical Nigeria Limited, Ikare-Akoko, Nigeria
}

Email address:

ayomide.agunloye@aaua.edu.ng (A. O. Agunloye), agunloyeei@yahoo.com (E. I. Agunloye), nathaniel.ajayi@aaua.edu.ng (N. O. Ajayi)

${ }^{*}$ Corresponding author

To cite this article:

Ayomide Oluwaseyi Agunloye, Emmanuel Idowu Agunloye, Nathaniel Oluwadusi Ajayi. Construction of a Simple USB Powered LED Light Source for Illuminating Optical Microscopes. American Journal of Embedded Systems and Applications. Vol. 8, No. 1, 2021 , pp. 7-11. doi: 10.11648/j.ajesa.20210801.12

Received: June 1, 2021; Accepted: June 21, 2021; Published: June 30, 2021

\begin{abstract}
Tungsten and halogen lamps are normally used for microscope illumination. However, if there is an electrical fault in any of these lamps, extra money to purchase a new one and the sacrifice of a long time for its arrival may be required to get the lamp replaced, especially in some developing countries. The aim of this study therefore is to develop a cheap and easily obtainable light source, built with Light Emitting Diodes (LEDs) to replace a faulty tungsten or halogen lamp. The LEDs are cheap to buy, easily available and operate at a much lower voltage. A light source, using LEDs, was designed and constructed to fit easily into the space previously occupied by the tungsten or halogen lamp. An integrated switching low power DC-DC converter, having a microcontroller unit at its heart and powered from a 5 Volt source, was also constructed to control the LED light source. It was observed that the contrast of the sample image obtained with the halogen lamp was reproduced by the LED light source. In this study, a replacement LED based light source for illuminating optical microscopes was constructed, and it gave a reasonable result compared to that obtained by using the halogen lamp. This work will be very useful in those places where it may be very difficult to get a quick replacement halogen or tungsten lamp or where electric power is not always available.
\end{abstract}

Keywords: Microscope, Light Emitting Diode (LED), Universal Serial Bus (USB), Optical Illumination, Halogen Lamp

\section{Introduction}

A laboratory optical microscope is a very useful tool for examining the structure of tissues, cells and microbes [1]. A good uniform and bright white light similar to sunlight is an essential part of optical microscopy [2], but requires electricity from the national grid or the local portable generator to generate it, thus making its use for a long time to be expensive.

Since microscopic examination takes very long hours to execute, many poor local schools and hospitals where electricity may not be available for a long time or often, may not be able to afford the cost of making many microscopic examinations for long hours.

Tungsten and halogen lamps are normally used for microscope illumination [3]. However, if there is an electrical fault in the lamp, an extra money and a long time may be needed or sacrificed to get the lamp replaced. Under this condition, a cheap and easily constructible LED based light source can be a quick replacement, instead of looking for an extra money to purchase a new replacement tungsten or halogen lamp and sacrificing a long time for its arrival. Therefore, it is necessary to look for an alternative illumination source that can be a quick replacement and which can be operated cheaply in the local area.

Since LEDs can easily be obtained locally, a simple light source that uses LEDs can therefore be constructed to obtain a USB powered LED based light source needed to replace the tungsten or halogen lamp in the optical microscope.

Some studies had been carried out to redesign the illuminating of optical microscopes. For example, Satory [4] developed an LED light source to replace the tungsten light 
source, using a resistive circuit to control the LEDs. However, this circuit consumes power and quickly depletes the $4 \times 1.5 \mathrm{~V}$ AA batteries that power it.

In their own work, Amar Vijai et al. [5], replaced the tungsten light source with an LED light source. However, the LED power supply used was the old microscope power supply which can still be affected by the incessant power outages in rural areas of the developing world.

In this work, a microcontroller unit was used to provide a Pulse Width Modulation (PWM) for the inexpensive LED array. The light intensity of this system could be adjusted by a potentiometer connected to the microcontroller. The LED array unit was a 5 Volt system and could be powered from any 5 Volt DC power source such as a Smartphone charger or a power bank. This unit will then serve as a replacement light source for classroom optical microscopes.

\section{Materials and Methods}

\subsection{Basic Concept}

Optical microscopes utilize light sources with adjustable brightness. The adjustment of the brightness is normally achieved via a resistive dimmer circuit; however, it does not provide a linear brightness graduation [6]. Light Emitting Diodes (LEDs) are current controlled devices and the relationship between their emitted light intensity and the current flowing through them is approximately linear at certain regions. This relationship is loosely dependent on the applied voltage, and thus the intensity of an LED light can be precisely controlled by varying the power applied to its terminal [7]. It should be noted that the intensity of an LED light is inversely proportional to its $\mathrm{P}-\mathrm{N}$ junction temperature which increases with the applied power. Therefore, to keep this temperature at a minimum value during high powered applications, the LED(s) must be mounted on heat-sinks [8].

\subsection{Pulse Width Modulation (PWM)}

Pulse width modulation (PWM) is an efficient way to vary the power delivered to a system using modern electronic power and semiconductor switches [9]. This principle is widely used in power electronics applications for controlling power converters (DC/DC and DC/AC) [10], and power switching applications for controlling analog circuits via digital outputs [11].

A typical basic PWM controlled Buck type (step-down) DC/DC converter [12] is shown in Figure 1.

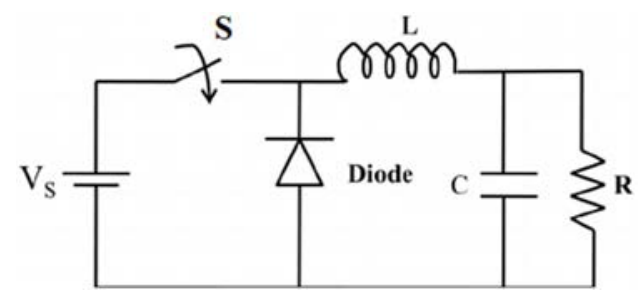

Figure 1. Buck type (step down) DC converter [13].
The PWM signal is generated by comparing an adjustable reference voltage, $V_{\text {ref }}$, with a triangular wave of constant amplitude and frequency. This frequency determines the switching frequency of the converter and it must be high enough so as not to affect the load.

The amplitude of the reference voltage $V_{\text {ref }}$ determines the duty cycle of the PWM signal (which is the ratio of the on time of one cycle of the PWM signal to its period). The relationship between the output voltage and the input voltage is shown below;

$$
V_{0}=D \times V_{\text {in }}=\frac{t_{\text {on }}}{T_{s}} V_{s}=\frac{V_{r e f}}{V_{t r}} V_{\text {in }}
$$

Where;

$V_{\text {in }}$ is the converter DC input voltage,

$D$ is the PWM signal duty cycle $(0 \leq D \leq 1)$,

$t_{\text {on }}$ is the PWM signal ON time,

$T_{S}$ is the converter switching period,

$V_{t r}$ is the triangular wave amplitude and,

$V S$ is the source voltage.

It should be noted that the longer the switch stays on, the higher is the power delivered to the load.

The power switch S is usually of MOSFET or IGBT type in high power applications but can also be replaced by transistors and monolithic ICs (in which the controller and switching elements are on the same chip) in low power applications [14-16]. PWM switching frequencies in these applications range from the $\mathrm{kHz}$ range to a few $\mathrm{MHz}$.

One major advantage of the PWM switching technology is the reduced power losses in the switching element. This is because when the switching element is off, no current practically flows through it, and when it is on, the voltage drop across it is low. Other advantages of PWM over primitive methods of power control are: the ease of control circuitry design and the ease of integration with digital control systems [17].

\subsection{PWM with Microcontrollers}

Microcontrollers are system-on-chip digital devices. They are only capable of working with discrete digital signals which are either HIGH and LOW with respect to their input voltage.

It is possible to achieve PWM by using software loops to create the PWM waveforms. Nevertheless, PWM generation in microcontrollers can be done using their on-board counters and timers. These timers and counters employ the hardware semiconductor registers and dividers that are directly integrated in the chip [18].

Consumer type microcontrollers are capable of generating PWM waveforms up to their clock frequencies divided by 256 The duty cycles of these waveforms can be completely varied between the fully off (0) and the fully on (1) states while the microcontroller is working, as control bits of the PWM register can be set and cleared at any time during the microcontroller operation. If required, the PWM frequency can be changed by dividing the microcontroller clock frequency by a pre-set pre-scalar factor [19]. 


\section{Circuit Diagram and Construction}

Table 1 contains the list of the parts used in the LED light source and their respective prices. As a replacement source, it was important that it should easily be installed. Thus, it was designed to fit easily into the space previously occupied by the halogen lamp. As halogen lamp and LED are controlled by different technologies, a new control system was designed for the LED array. The control unit was an integrated switching low power DC-DC converter with a microcontroller unit (MCU) at its heart.

Table 1. Components used and their prices.

\begin{tabular}{ll}
\hline COMPOENTS & PRICE (US Dollars) \\
\hline Arduino Nano & 3.0 \\
Variable resistor & 0.2 \\
Fixed value resistor & 0.2 \\
BC547 & 0.5 \\
TIP120 & 0.5 \\
White LED (5) & 0.5 \\
Casing & 3.6 \\
Total & 8.5 \\
\hline
\end{tabular}

In this study, the Arduino Nano prototyping board was used because of its compactness. The Arduino Nano was configured to produce a PWM waveform at a frequency of 490 Hertz from pin 3. This waveform was fed to the small signal transistor Q2 which was used to isolate the MCU and provide adequate base drive current to the switching transistor Q1 of the system.

The switching transistor Q1 and the LED array load were connected in a buck converter configuration. The LC low pass filter was replaced by the RC low pass filter formed by the current limiting resistor $\mathrm{R} 3$ and the internal capacitance of the LED array load. The duty cycle of Q1 was adjusted, using the potentiometer RV1 that was connected to the 'Analog to Digital' converter (ADC) of the Arduino Nano. This potentiometer was connected as a voltage divider. It could be set in the clockwise (CW) direction to increase the duty cycle, or in the anticlockwise direction to decrease it. Figure 2 shows the block diagram of the LED light source.

The control unit was powered from a 5 Volts USB power source, and housed in a separate casing outside the microscope. This allowed it to be easily detached from the LED array unit installed in the microscope and it facilitated the easy movement of the whole system. Figure 3 shows the circuit diagram of the control unit.

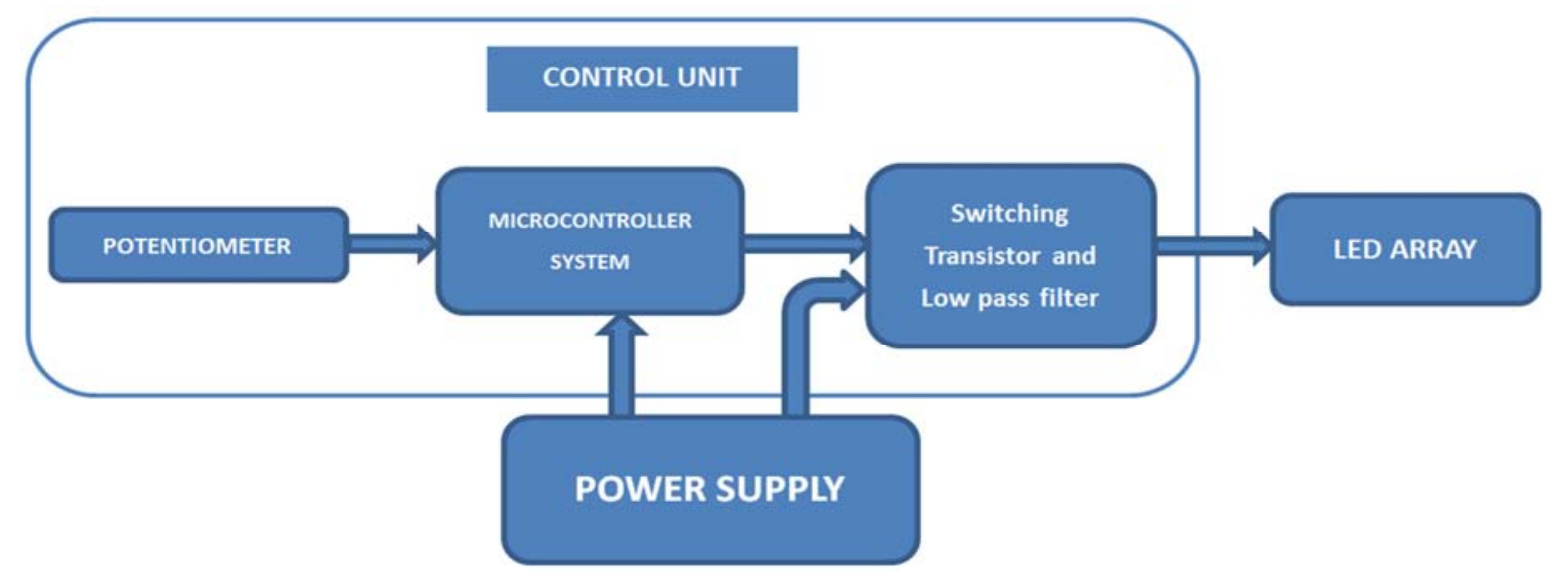

Figure 2. Block diagram of the LED array system.

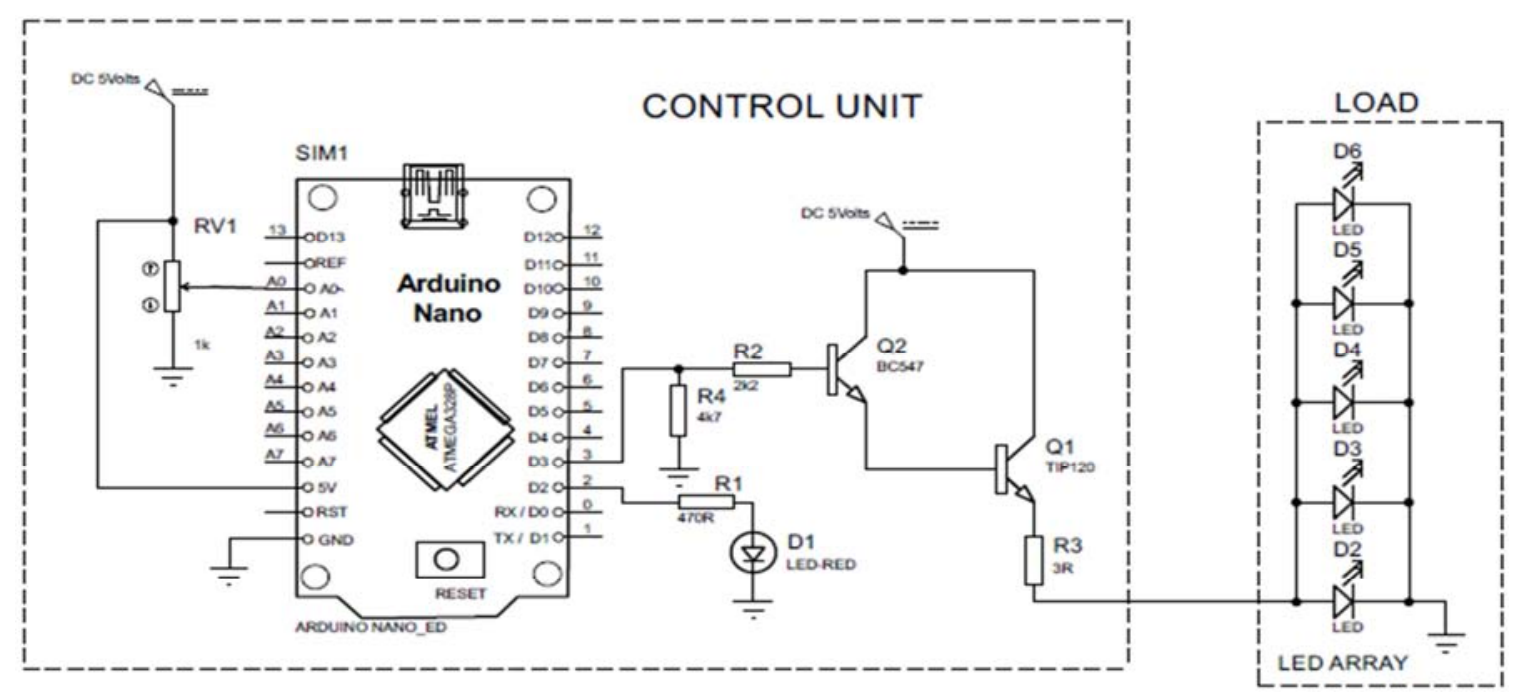

Figure 3. Circuit diagram of the control unit. 


\section{Results and Discussion}

To compare the performance of the LED light source with that of the halogen lamp, a slide of the Leishman stain of blood sample was captured with a generic USB microscope camera, first with the optical microscope having a halogen lamp as the light source and then with the microscope having the LED as the light source. Figure 4 shows the slides of Leishman stain obtained with Halogen lamp (SLIDE A) and then with the LED light source (SLIDE B).

It was observed that the contrast of the sample image achieved with the halogen lamp was reproduced by the LED light source.

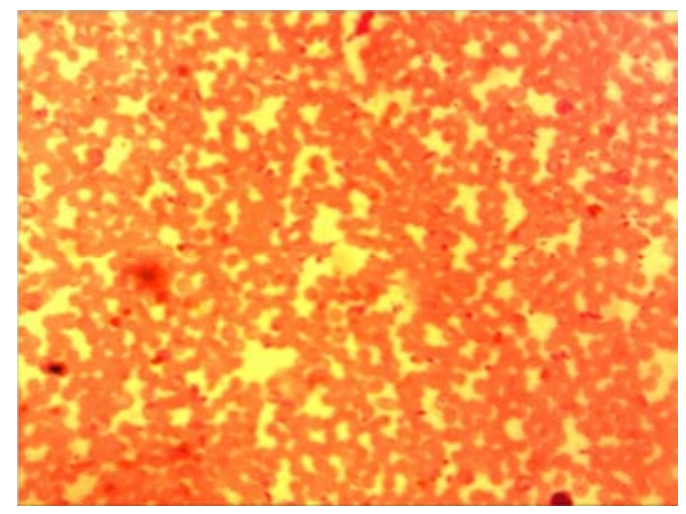

(SLIDE A)

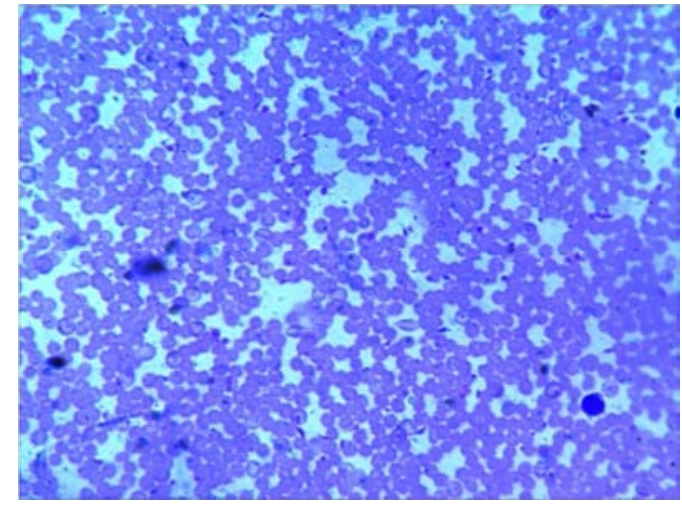

(SLIDE B)

Figure 4. Slides of images of Leishman stain obtained with; (SLIDE A) Halogen lamp and, (SLIDE B) LED array system. Scale bar:200 $\mu \mathrm{m}$

While the image of the sample, obtained with the halogen lamp showed a richer composition of broad-spectrum with noticeable red-shift, the image with the LED light source had a slightly narrow spectrum, making it to be sharper than the image obtained with the halogen lamp.

It was noticed that setting a desired brightness was easier with the LED light source than with the halogen lamp, and that the fluctuations in the mains power had effect on the brightness of the halogen lamp but not on the brightness of the LED light source.

In terms of the measured consumed power at maximum brightness, it was observed that the halogen lamp consumed about twenty-five (25) times more power than the LED light source (Figure 5), making the LED light source to be more economical and energy efficient than the halogen lamp.

\section{Power Consumption at Maximum Brightness}

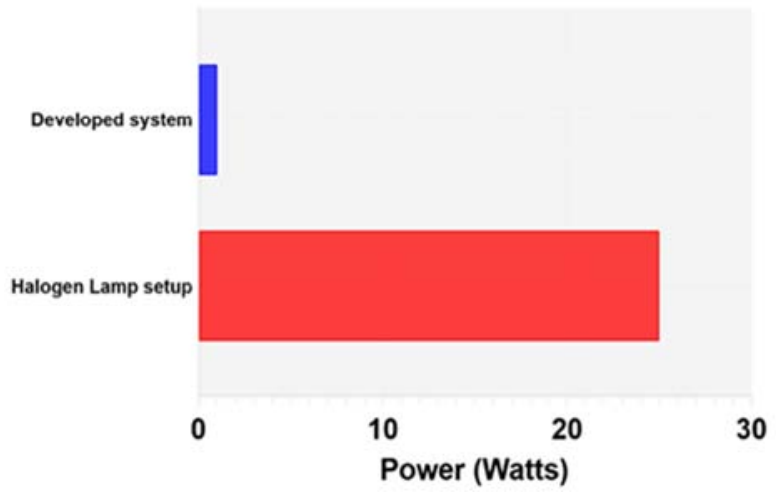

Figure 5. Graph of Power consumption for the two setups.

Other researchers have tried to address the cost of illumination in conventional microscopes by using LED light sources, but they have not completely solved the problem of power consumption in their modification and the problem of incessant power outages in rural areas of the developing world.

In general, the ultimate limit to the resolution of a light microscope is set by the wavelength of the visible light which ranges from about $400 \mathrm{~nm}$ for violet, to $700 \mathrm{~nm}$ for deep red [20], and makes it possible to observe bacteria and mitochondria which are about $500 \mathrm{~nm}$ wide. Therefore, any light source with wavelengths in this range can be used for optical microscopy. Because LEDs emit light with a relatively narrow wavelength band, the size of particles that can be detected will be limited by this, as the smallest particle detectable will depend on the smallest wave length of the light band.

It is noted that the color of the light within the visible range emitted by different LEDs may be different from one another. However, there are some LEDs that can emit a visible radiation close to white light. In the absence of such types, a close to white light illumination can be obtained by having a combination of LEDs that are available in the surrounding.

\section{Conclusion and Recommendation}

In this study, a replacement LED array lighting unit for a tungsten or halogen lamp in an optical microscope had been constructed. This new illuminating unit gave reasonable and comparable result to those obtained by using the halogen lamp. The specimens illuminated with it were easily observable. The layout diagram of the LED power source in this work is easy to follow and to construct. The components are easily available and very cheap, making the constructed LED light source to be a quick and an inexpensive replacement of the imported light source of the microscope.

Since the condenser lens used to collimate the light rays from the LEDs was originally built for the halogen lamp, the precise positioning of the LED array unit will be required to ensure that the light rays from the LED light source diverge as 
little as possible. Redesigning a new condenser exclusively for the LED array unit will therefore, further reduce diffraction and give a better sample illumination.

\section{References}

[1] OpenLearn, "Introduction to microscopy: Conclusion," Open University, 2021. https://www.open.edu/openlearn/science-maths-technology/bi ology/introduction-microscopy/content-section-7 (accessed Apr. 26, 2021).

[2] Labcompare, "Microscope Light Sources," 2017. https://www.labcompare.com/Microscopy-and-Laboratory-Mi croscopes/184-Microscope-Light-Sources/ (accessed Apr. 26, 2021).

[3] M. W. Davidson, "Tungsten-Halogen Incandescent Lamps," ZEISS Microscopy Online Campus, 2021. $\mathrm{http}: / /$ zeiss-campus.magnet.fsu.edu/articles/lightsources/tungst enhalogen.html (accessed Apr. 26, 2021).

[4] C. Sartory, "LED lighting for microscopes," Quuekett J. Microsc., vol. 40, pp. 701-711, 2008.

[5] N. Amar Vijai, O. Ika Kustiyah, F. Arfan Eko, M. Eko, Suhartono, and N. Galih, "Development of a Low Cost and Calibrated Digital Microscope with Replaceable LED's Light Source Basedon ImageJ for Microscopic Surveillance," Int. J. Eng. Res. Ind. Appl., vol. 5, no. Iv, pp. 189-198, 2012.

[6] M. Enns, "Thermal radiation - Light Intensity vs Power for a Small Incandescent Lamp," Physics Stack Exchange, 2019. https://physics.stackexchange.com/questions/470595/light-inte nsity-vs-power-for-a-small-incandescent-lamp (accessed Nov. $08,2020)$

[7] S. King, "Luminous Intensity of an LED as a Function of Input Power," ISB (International Sch. Bangkok) J. Phys., vol. 2, no. June, pp. 1-4, 2008.

[8] T. Sato and V. N. Murthy, "Light-emitting diodes for biological microscopy," Cold Spring Harb. Protoc., vol. 7, no. 12, pp. 1211-1222, 2012, doi: 10.1101/pdb.top072306.

[9] J. Holtz, "Pulsewidth modulation-a survey," in PESC 92 Record. 23rd Annual IEEE Power Electronics Specialists Conference, 1992, vol. 39, no. 5, pp. 11-18, doi: 10.1109/PESC.1992.254685.
[10] N. Mohan, T. M. Undeland, and W. P. Robbins, Power electronics: converters, applications and design. John wiley \& sons, 2003.

[11] M. Barr, "Pulse width modulation," Embed. Syst. Program., vol. 14, no. October, pp. 103--104, 2001.

[12] N. González-Fonseca, J. De León-Morales, and J. Leyva-Ramos, "Observer-based controller for switch-mode DC-DC converters," Proc. 44th IEEE Conf. Decis. Control. Eur. Control Conf. CDC-ECC '05, vol. 2005, pp. 4773-4778, 2005, doi: 10.1109/CDC.2005.1582916.

[13] S. Lakshmi and T. S. R. Raja, "Design and implementation of an observer controller for a buck converter," Turkish J. Electr. Eng. Comput. Sci., vol. 22, no. 3, pp. 562-572, 2014, doi: 10.3906/elk-1208-41.

[14] R. Miftakhutdinov, A. Nemchinov, V. Meleshin, and S. Fraidlin, "Modified asymmetrical ZVS half-bridge DC-DC converter," in APEC '99. Fourteenth Annual Applied Power Electronics Conference and Exposition. 1999 Conference Proceedings (Cat. No. 99CH36285), 1999, vol. 1, pp. 567-574 vol. 1, doi: 10.1109/APEC.1999.749737.

[15] Jung-Hui Cheng, A. F. Witulski, and J. L. Vollin, "A small-signal model utilizing amplitude modulation for the class-D converter at fixed frequency," IEEE Trans. Power Electron., vol. 15, no. 6, pp. 1204-1211, Nov. 2000, doi: $10.1109 / 63.892835$.

[16] E. Koutroulis, A. Dollas, and K. Kalaitzakis, "High-frequency pulse width modulation implementation using FPGA and CPLD ICs," J. Syst. Archit., vol. 52, no. 6, pp. 332-344, 2006, doi: 10.1016/j.sysarc.2005.09.001.

[17] S. K. Singh et al., "A Survey and Study of Different Types of Pwm Techniques Used in Induction Motor Drive," J. Eng. Sceince Adv. Technol., no. 4 (1), pp. 18-22, 2014.

[18] L. Loflin, "Pulse Width Modulation Microcontroller Power Control," "Pulse Width Modulation Microcontroller Power 2018. http://www.bristolwatch.com/ele/pulse_width_modulation.htm (accessed Nov. 08, 2020).

[19] Microchip, “ATmega328P - 8-bit AVR Microcontrollers,” 2017. https://www.microchip.com/wwwproducts/en/ATmega328p (accessed Dec. 23, 2020).

[20] Science Learning Hub, "Magnification and resolution," 2012. https://www.sciencelearn.org.nz/resources/495-magnificationand-resolution (accessed Apr. 26, 2021). 